\title{
Evolución de la eliminación de ooquistes de Cryptosporidium sp. en las heces de niños con síndrome diarreico
}

\author{
Renzo Tassara O. ${ }^{1}$; Juan Carlos Weitz V.2, ${ }^{2}$; Antonio Atías M. ${ }^{1}$ \\ Cryptosporidium sp. duration of oocysts excretion \\ in children with acute diarrhea
}

\begin{abstract}
A prospective follow up was done to 02 children $(2$ to 40 months of age) who were admilted to hospital because of Cryptosporidium sp. Infection and acule diom to to measure the duration of fecal oocysts excretion. Fecal samples were laken each two days and stained with Zlehl Neelsen technique. In 46 children a complete survey was done until oocysts disappeared from iheir faeces. Sixteen children were discharged from hospital before completion of the sludy and they didnt corne back lo follow up so they were not considered tor this report. The average duration of cocysls excretion, from diognosis to the last positive fecal somple, wos 10 days (ronge 3 to 48), In 22 cases $(47.8 \%$, excretion was longer than 15 days, and in $32109.6 \%$, 00cysls persisted in faeces after clinlcal recovery. No patients evolved to chronic persistenl infection. The long protozoal excretion lime in osymptomatic convolescent patients is of epidemiclogical significance for the spread of this infection.
\end{abstract}

IKey words: Cryplosporidium, fecal excretion, diartheo, infonile.]

Cryptosporidium sp. ha sido ampliamente aceptado como uno de los agentes etiológicos de diarrea aguda más importantes en la edad pediátrica. Entre los organismos parasitarios sería el más frecuente, en el total de patógenos ocuparía el cuarto o quinto lugar -después de Rotavirus, $E$. coli enteropatógena y Campylobacter-, siendo similar en importancia relativa que Shigella sp., ${ }^{1,2}$.

Por lo general, en las diarreas infecciosas la desaparición đel agente etiológico en las muestras fecales precede o es concomitante a la mejoría sintomática, excepto casos en que el paciente mantiene portación asintomática crónica. Este parásito afecta a las personas inmunocompetentes de manera autolimitada, provocando un trastorno gastrointestinal agudo. Sin embargo, en pacientes inmunocomprometidos la evolución

1. Unidad de Parasitología, División Ciencias Médicas Occidente. Fecultad de Medicina, Universidad de Chile.

2. Laboratorio de Referencia Parasitología, Instituto de Salud Pública.

3. Departamento de Medicina, División Ciencias Médicas Occidente, Facultad de Medicina, Universidad de Chile. tiende a la cronicidad, con eliminación de ooquistes en cantidad variable, pero mantenida en el tiempo y acompañada de síntomas ${ }^{3-5}$. Se han descrito algunos casos de infecciones aparentemente asintomáticas y transitorias por Cryptosporidium sp. pero no infecciones asintomáticas persistentes $^{6-8}$.

En un estudio anterior observamos que niños con crytosporidiosis intestinal habian continuado eliminando ooquistes en las heces varios días después de la mejoría clínica. El hecho que un convaleciente de cryptosporidiosis persista eliminando ooquistes tiene gran importancia, puesto que estos elementos son inmediatamente infectantes, de fácil transmisibilidad, muy resistentes a las condiciones ambientales y a los desinfectantes de uso habitual, al mismo tiempo que la dosis infectante requerida sería muy pequeña, habiéndose observado transmisión experimental con sólo 10 ooquistes?.

El propósito de este trabajo es describir la evolución de la eliminación de ooquistes de Crypiosporidium sp. en las heces de niños con diarrea, cuando en ellas se identificó el agente al comienzo de la enfermedad. 


\section{Material y Método}

En el transcurso de 18 meses se estudiaron, en forma prospectiva, 62 niños de 2 a 40 meses de edad (tabla 1). hospitalizados en el Servicio de Pediatria del Hospital San Juan de Dios, provenientes del área occidente de Sanliago. Todos cursaban con diarrea y se había demostrado Cryptosporidium sp. en sus heces, mediante la técnica de Ziehl Neelsen modificada ${ }^{10}$, ya sea corno agente único o asociado a otros enteropatógenos.

Desde el momenio en que se demostro la existencia de Cryptosporidium sp. en las deposiciones, a cada paciente se le recolectaron muestras fecales en fijador PAF, aproximadamente cada dos días, las que futron procesados por el método de Burrows, confeccionando un extendido que se tin̂ó con Ziehl Neelsen. La leciura de los frous feçles se efectuó mediante microscopio de luz con aumento $1000 \mathrm{x}$, recorriendo la muestra completa. Además, se confeccionó una ficha individual que contenía los datos generales del paciente y su evolución clínica. En los casos en que el alta del hospital se dio antes que la negativización parasitológica, los pacientes fueron mantenidos en control ambulatorio, en el policlínico de la especialidad, hasıa la desaparición de los oqquistes en al menos dos exánenes consecutivos. Para ello se entreg6 a cada paciente el número necesario de frascos con fijador y la indicación de tomar muestras cada dos días (especificando la fecha debidamente).

\section{Resultados}

De los 62 pacientes, $46(74,2 \%)$ fueron seguidos hasta la mejoría parasitológica. Los 16 restantes $(25,8 \%)$ se descartaron del estudio debido a que, si bien al alta hospitalaria aún eliminaban ooquistes, no concurrieron al control ambulatorio indicado y fue imposible recupcrarlos mediante visita domiciliaria, porque los datos consignados en la fichas correspondían a direcciones falsas. Se efectuaron, en total, 455 exámenes coproparasitológicos de seguimiento, promedio 7,3 por niño, variación 4 a 24 . El período control fluctuó de 4 a 78 días por paciente, promedio 21,1 días.

En la tabla 2 se observa que la duración tolal de la eliminación de ooquistes en los 46 casos, desde el momento del diagnóstico hasta la desaparición de los elementos parasitarios, fue como promedio 16 , márgenes 3 a 48 , días. La mayoría de los casos, 33 pacientes $(71,7 \%)$, eliminaron Crypiosporidium sp. durante 6 a 20 días. En 22 $(47,8 \%)$ casos se comprobó excreción de ooquistes por más de 15 días.

La cantidad de elementos parasilarios eliminados fue disminuyendo paulatinamente durante el período sintomático, pero en $32(69,6 \%)$ casos se registró persistencia de la excreción de 00 quistes después te terminada la diarrea. La figu-
Tabla 1

Distribución por edad de 46 pacientes con Cryptosporidium sp.

\begin{tabular}{crr}
\hline Ed2d (meses) & n & \% \\
\hline 0.6 & 20 & 43,4 \\
7.12 & 17 & 37,0 \\
$13-18$ & 7 & 15,2 \\
31.36 & 1 & 2,2 \\
37.42 & 1 & 2,2 \\
\hline Total & 46 & 100,0 \\
\hline
\end{tabular}

Tabla 2

Período de eliminación de ooquistes de Cryptosporidium sp. entre el primer y último examen positivo

\begin{tabular}{crr}
\hline $\begin{array}{c}\text { Duración positiva } \\
\text { (dias) }\end{array}$ & n & Pacientes \\
\hline$<5$ & 3 & 6,5 \\
$6-10$ & 11 & 23,9 \\
$11-15$ & 10 & 21,7 \\
$16-20$ & 12 & 26,0 \\
$21-25$ & 5 & 11,0 \\
$26-30$ & 4 & 8,7 \\
$310>$ & 1 & 2,2 \\
\hline Total & 46 & 100,0 \\
\hline
\end{tabular}

ra 1 muestra el número de niños y la duración en días de la infección, una vez normalizadas las deposiciones. De los 32 casos, $20(62,5 \%)$ seguian climinando ooquistes a los siete días; $4(12,5 \%)$ a las dos semanas y sólo uno después de la tercera semana, hasta los 42 días. Ningún paciente evolucionó, durante el período de estudio, hacia una infección crónica persistente.

Cinco nifos registraron una muestra negativa durante el período de eliminación de ooquistes, hecho confirmado al repetirla con controles de tinción, siendo positivas las muestras precedentes y las posteriores. En tres de estos casos el fenómeno sucedió poco antes de la desaparición de los ooquistes en las heces, pero en los dos restantes, durante un período de alta eliminación de elementos parasitarios en las deposiciones.

Si bien no se contaron los elementos en los frotis, en todos los niños estudiados se observó disminución progresiva de Cryptosporidium sp. hasta su desaparicion. 


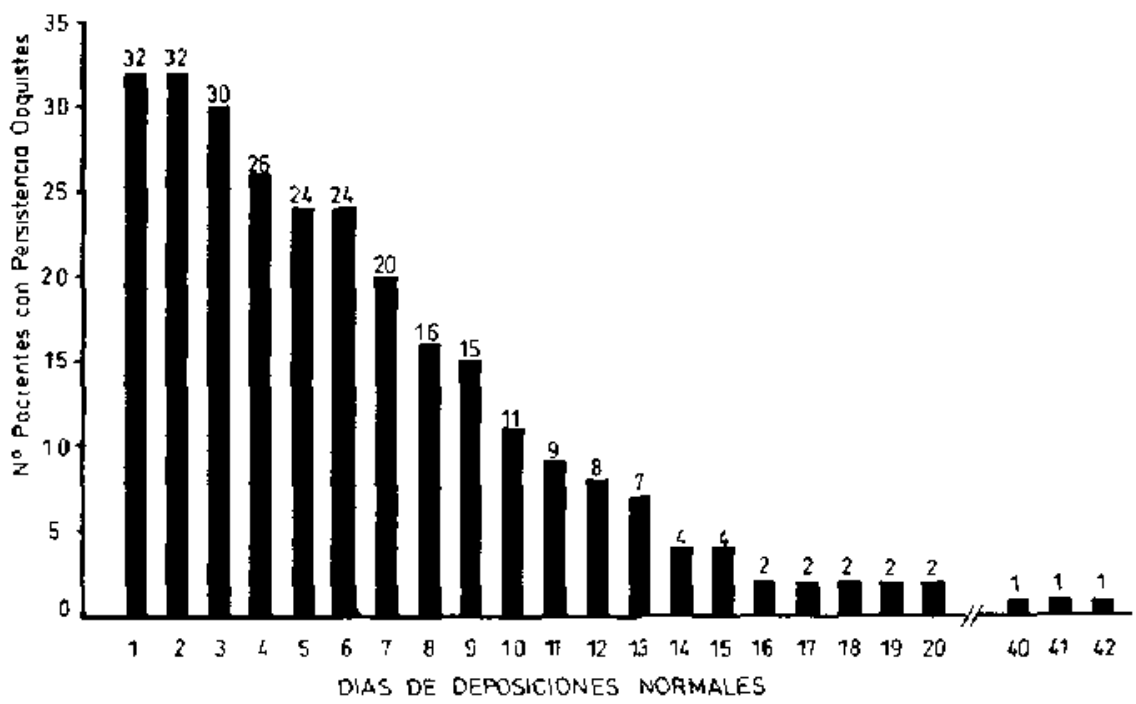

Figura 1: Duración de la elìninación de ooquistes de Cryptosporidium $s p$, en niños convalecientes de diarrea.

\section{Comentario}

Pocos estudios analizan la evolución de la eliminación de ooquistes de Cryptosporidium sp. durante y después de un episodio de diarrea aguda. En la mayoría de las experiencias, el seguimiento coproparasitologico completo se ha efectuado en un reducido número de casos o éstos corresponden a brotes epidémicos. Nuestros resultados sobre la duración de la excreción de ooquistes, a partir del diagnóstico de la infección, son concordantes con los de otras publicacione $\mathrm{s}^{1 \mathrm{l}-13}$.

La persistencia de la eliminación de Cryptosporidium sp. en las heces, posterior a la mejoría clínica, ha sido comunicada en varios estudios con duraciones de 21 a 60 dias $^{11-13}$. En nuestra casuística, en más de dos tercios de los riños se mantuvieron positivos los exámenes de Ziehl Neelsen, luego de la normalización de las heces, hasta 42 dias.

La alta transmisibilidad de este parásito ha sido sugerida en varias publicaciones: en brotes epidémicos institucionales, infección de contactos intrahospitalarios y experimentales ${ }^{14-18}$; en nuestros estudios hemos observado infección de contactos familiares e institucionale ${ }^{19,} 20$. La portación prolongada en los convalecientes de esta parasitosis favoreceria la diseminación, si además se consideran la alta resistencia de los ooquistes a las condiciones ambientales y a los detergentes de uso habitual, sin olvidar la baja dosis infectante?, 21. Por otra parte, este estado de "portador asintomático" scría, aparentemente, transitorio en todos los casos, ya que ningún paciente continuó eliminando indefinidamente ooquistes en sus heces.

A la luz de estos antecedentes y ante la importancia de esia parasitosis, demostrada en varias publicaciones nacionales ${ }^{2-24}$, es perentorio enfatizar el cuidado en los hábitos higiénicos de todo convaleciente de cryptosporidiosis, así como también recomendar medidas especiales en adultos cuya actividad se relacione con la manipulación de alimentos y la atención de pacientes.

Otras coccidiosis, como isosporosis y sarcocystosis, evolucionan, como la cryptosporidiosis, en forma autolimitada, pero se diferencian de ella en que la desaparición de los elementos parasitarios es concomitante a la mejoría clínica.

La investigación farmacológica en el tratamiento de la cryptosporidiosis no ha dado los frutos esperados, por lo que hasta la fecha no se dispone de un medicamento probadamente efectivo. Es por ello que la estrategia más adecuada para el control de esta infección debiera estar orientada a la prevención, enfatizando en el manejo sanitario del paciente infectado, con especial acento en la profilaxis del paciente con deficiencias inmunitarias. 
Se concluye que en la mayoría de los pacientes con cryptosporidiosis persiste la eliminación de ooquistes por períodos prolongados, después de la mejoría clínica, y ello constituiria un hecho epidemiológico significativo en la propagación de esta infección.

\section{Resumen}

Se efectuó seguimiento parasitológico en las deposiciones, mediante tinción de Ziehl Neelsen, de 62 niños de 2 a 40 meses de edad, que fueron hospitalizados por diarrea aguda, en los que se había demostrado Cryptosporidium sp. en sus deposiciones. En 46 casos se logró mantener la vigilancia hasta la mejoría parasitológica. Los restantes 16 se descartaron por abandono del control. La duración promedio de excreción de ooguístes desde el diagnóstico hasta el último examen positivo fue de 16 dias ( 3 a 48 días). En $22(47,8 \%)$ ninos la excreción se prolongó más de 15 dias y en 32 casos $(69,6 \%)$ los ooquistes persistieron luego de la mejoría clínica, hasta 42 dfas. Ningún paciente evolucionó hacia una infección crónica persistente. La eliminación prolongada en el tiempo de este protozoo en los infectados constituye un factor epidemiológico significativo en la propagación de esta infección, por lo que se deben reforzar las medidas sanitarias no sólo en el paciente sintomático sino que durante la convalecencia.

(Palabras clave: criptosporidiosis, diarrea aguda, eliminación fecal de ooquistes.)

\section{Referencias}

1. Baxby $D$, Hart $C$ : The incidence of cryplosporidiosis: a two year prospective survey in a children's hospital. J Hyg Camb 1986; 96: 107-111.

2. Mata L. Botaños H, Pizarro D et al.: Criptosporidiosis in children from highland Costa Rics ruraf and urban areas, Am J Trop Med Hys 1984; $33: 24-29$.

3. Hart C, Baxby D, Blwadell N: Gastroenteritis due to Cryptosporidium: a prospective survey in a children's hospital. I Infection 1984; 9: 264-270.

4. Petersen $C$ : Cryptosporidiosis in patients infected with the human immunodeficjency vinus. Clin Infect Dis 1992; 15: 903-909
5. Pitlik S, Fainstein V. Garza D, et al.: Human cryptosporidiosis: spectrom of disease. Arch Intern Med 1983; 143: 2269-2275.

6. Vuorio A, Jokipial $M$, Jokipili L: Cryptasporidiwn in asymptomatic children. Rev Infect Dis 1991; 13: 261264.

7. Crawford F, Vermund S, Ma J, Deckelbaum $R$ : Asymptomatic cryptosporidiosis in a New York city day care centet. Pediatr Infect Dis J 1988; 7: 806-807.

8. Zar F: Asymptomatic carrier state for Cryplosporidium. Amer J Med 1990; 89: 545-546.

9. Miller R, Bronsdon M. Morton W: Experimental cryptosporidiosis in a primate model. J Infect Dis 1990; 161:312-315.

10. Garcia $L$, Bruckner $D, B r e w e r ~ T e t$ at.: Techuiques for the recovery and identification of Cryptosporidium oocysts in faeces. J Hyg Camb 1984; 92:317-323.

11. Stehr-Green J, MCCaig L, Remsen $H$, et al.: Shedding of ocysts in immunocompetent individuals intected with Cryptosporidtun. Am J Trop Med Hyg 1987; 36: 338342.

12. Baxby D, Hart C, Blundell N: Shedding of oocysts by imunocompetent individuals with cryptosponidiosis. $J$ Hyg Canb 1985; 95: 703-709.

13. Jokipü $L$, Jokipii A: Timing of symptoms and cocysts excretion in human cryptosporidiosis. N Engl J Med 1986; 315: 1643-1647.

14. Nawarrete S, Stetier H. Avilo C, et al:: An outbreak of Cryptasporidium diarthea in a pediatric hospital. Pediat Infect Dis I 1991; 10: 248-250.

15. Rawn P, Lundgren J, Kjaeidgaord P, et at.: Nosocomial outbreak of cryptosporidiosis in AIDS patients. Br Med J 1991; 302: 277-280.

16. Koch $K$, Phillips $D$, Aber R, et al.: Cryptosporidiosis in hospital personnel. Ann Interm Med 1985; 102: 593 . 596.

17. Smish $H$, Rose J: Waterborne cryptosporidiosis. Parasitol Today 1990; 6: 8-12.

18. Joce $R$, Bruce $J$, Kiely $D$, et al: An outbreak of cryptosporidiosis associaled with swimming pool. Epidemiol Infect 1991; 107: 497-508.

19. Weitz JC. Tassora $R$, Mercado $R$, et al.: Brote de cryptosporidiosis en un centro de recuperación notricional. Rev Chil Pediatt 1987; 58: 50-53.

20. Weitz JC, Tessara $R$, Fabres J: Incidencia de Crypiosporidium sp en niños con diarrea aguda de un jardín infantil del área occidente de Santiago. Parasitol al Día 1992: 16: 44-46.

21. Fayer R, Ungar B; Cryptosporidium and cryptosporidiosis. Microbiol Rev 1986; 50: 458-483.

22. Tassara $R$, Weitz JC, Mercado $R$, Espinoza M, Atías A; Cryptosporidiosis humana: características clinicas en 11 casos. Bol Hosp S Juan de Dios 1986; 33; $237-40$.

23. Weitz JC, Mercado $R$, Tassara $R$, et al.: Frecuencia de Cryplosporidiun sp. en pacientes hospitalizados con síntomas digestivos. Rev Chil Pediatr 1986; 57: 10-12.

24. Weitz JC. Tessare R, Mercodo R: Cryplosporidiosis in chilean children. Trans R Soc Trop Med Hyg 1988; 82: 335. 